\title{
CAMINOS PARALELOS INCAICOS EN MAPOCHO NORTE, CHILE
}

\author{
INCA PARALLEL ROADS IN NORTH MAPOCHO, CHILE
}

\author{
Rubén Stehberga, Gonzalo Sotomayor ${ }^{B}$, Claudia Prado $^{c} \&$ Carolina Gatica
}

In memoriam Gonzalo Javier Sotomayor Cabeza

(17 febrero 1974-03 marzo 2015)

Se analizan dos mapas coloniales (1611) que proporcionan información con respecto a las características geográficas, a la propiedad europea de tierras, a los asentamientos indígenas y a la vialidad existente en la porción norte del valle del Mapocho. Uno de ellos ilustra el trazado de dos caminos paralelos denominados "camino antiguo del Inga" y "otro camino del Inga”. A través de reconocimientos en terreno (2014), uso de documentación colonial y cartografía histórica se logra identificar gran parte de sus trazados originales, incluyendo una reconstrucción cartográfica sig de las vialidades coloniales e incaicas.

Palabras clave: Qhapaq Nan, Caminos del Inca, Mapocho norte, Santiago, siglo XVI.

Two colonial maps (1611) are analyzed here, which provide information regarding geographic features, European land ownership, indigenous settlements and existing roadways in the northern area of the Mapocho valley. One of these maps depicts the layout of two parallel paths called "camino antiguo del Inga" and "otro camino del Inga" ('Inca's ancient road' and 'another Inca road', respectively). By means of field surveys (2014), and the use of colonial documentation and historical cartography, it has been possible to identify a large section of the roads' original tracings, including a GIS cartographic reconstruction of the colonial and Inca roads.

Keywords: Qhapaq Nan, Inca roads, North Mapocho, Santiago, 16th Century.

\section{INTRODUCCIÓN}

Este artículo trata de la presencia del Qhapaq Ñan en el acceso norte al valle del Mapocho. Después de analizar la historia de su investigación, su importancia, los problemas con la determinación de su trazado se centra en el estudio de dos mapas coloniales de 1611, cuya información pudo ser contrastada en terreno, lo que permitió identificar gran parte del trazado que tuvo este sistema vial prehispánico pese a que su evidencia física ha desaparecido. ${ }^{1}$

A fines del siglo xix, el archivero de la Biblioteca Nacional, Justo Abel Rosales, descubrió en el archivo de la Real Audiencia de Santiago un extenso juicio colonial datado en 1611, que trató de la exacta localización del camino de Chille o del Inga, el que servía de deslinde a las principales propiedades localizadas al norte de la ciudad de Santiago. Debido a que este camino estaba siendo cerrado, al tiempo que se abrían otros, se iniciaron litigios a los que concurrieron numerosos testigos, algunos de ellos indígenas del Perú que llegaron al valle del "Mapuche" a través de esta vía, acompañando a los primeros conquistadores europeos. De acuerdo

A Rubén Stehberg, Jefe del Área de Antropología. Museo Nacional de Historia Natural, Chile, email: Stehberg.ruben@gmail.com

B Gonzalo Sotomayor, Historiador.

c Claudia Prado, Licenciada en Arqueología. Coordinadora Centro Nacional de Sitios del Patrimonio Mundial, Subdirección Nacional de Gestión Patrimonial, Dibam, Chile, email: Claudia.prado@sngp.cl

D Carolina Gatica, Licenciada en Arqueología y Abogada. Consejo de Monumentos Nacionales, Chile, email: cgatica@monumentos.cl Recibido: diciembre 2015. Aceptado: abril 2016. 
a un informante, este camino venía de Aconcagua y Colina y descendía al valle de "Guachuraba". Dejaba al poniente (sic oriente) el cerro Pan de Azúcar y "después de hacer una extensa curva al este, llegaba a las rucas del cacique Huechuraba inclinándose al occidente, desde cuyo último punto continuaba en línea recta al río Mapuche" (Rosales 1887: 5-6), siguiendo el trazado de la calle Cañadilla, actual avenida Independencia, en el sector de la Chimba. El autor es perentorio al señalar que este camino era antiquísimo y fue abierto por los naturales mucho antes del arribo de los Incas, quienes lo reutilizaron.

Pese a que este libro fue editado con otro título a mediados del siglo xx (Rosales 1948), pasó desapercibido para la mayoría de los arqueólogos y etnohistoriadores que trabajaron el tema de la ocupación inca de Chile central y del valle del Mapocho-Maipo durante la segunda mitad del siglo xx. No fue mencionado por Keller (1960) en sus investigaciones incaicas de Quillota, ni por Mostny $(1947,1957)$ en sus estudios del cementerio incásico de La Reina y el adoratorio de cerro El Plomo, ni por Stehberg (1976) en su excavación del pucará de Chena, ni por Silva (1977-1978) en sus reflexiones sobre la presencia inca en el valle del Mapocho. Este último señaló que era "significativo que el Camino del Inca, monumento vial indispensable para el funcionamiento de la estructura estatal, sólo puede reconocerse con relativa seguridad hasta el río Copiapó" (Silva 1977-1978: 236). En la década de los ochenta se desarrolló un proyecto de reconocimiento de los Caminos del Inca en el Norte Chico y centro del país que permitió la identificación de varios segmentos de senderos longitudinales y transversales atribuibles al Tawantinsuyu. Un tramo bien identificado correspondió al de Alicahue Adentro y Almendrillo, entre los valles de Aconcagua por el sur y Choapa por el norte (Stehberg \& Carvajal 1988 a y b), con localización del tambo Conchuca (Stehberg et al. 1986). Estos estudios mencionaron, de manera muy vaga y sin lograr determinar su trazado, que el Camino Inca Longitudinal Andino seguía al sur por el valle de Putaendo, cruzaba la cuesta de Chacabuco, Colina La Vieja, portezuelo de Huechuraba, actual Avenida Independencia para dirigirse a las inmediaciones de Angostura de Paine, por el sur, sin lograr identificar físicamente sus restos.

En julio de 1980, Mario Rivera y John Hyslop realizaron una acuciosa prospección del área al norte de Santiago, para "proporcionar un ejemplo del
Camino del Inca en el extremo más meridional del imperio" (Rivera \& Hyslop 1984: 109). Conocieron la información proporcionada por las publicaciones de Justo Abel Rosales, a quien dieron crédito pese a ser un historiador aficionado. Desde el río Mapocho hasta el portezuelo o Pasos de Huechuraba, concordaron con la ruta propuesta por Rosales, pero desde este punto hacia el norte, el trazado de la ruta se complicó por falta de evidencia. El camino se dirigía "directamente al norte hacia el pueblo de Colina. En gran parte recorre el mismo trazado de la moderna Ruta 57 [...] Una fila de árboles delimita una línea recta al norte del Portezuelo" (Rivera \& Hyslop 1984: 116). El camino pasaba cerca del cerro Guaca, localizado $3 \mathrm{~km}$ al noreste del moderno pueblo de Colina, ascendía por un portezuelo de los cerros de Colina y $7 \mathrm{~km}$ al norte coincidía con la actual Ruta 57, siguiendo por el mismo trazado hasta la hacienda de Chacabuco y de allí al paso de $1.280 \mathrm{msnm}$ que separa los valles de Aconcagua del Mapocho.

En 2013, el historiador Gonzalo Sotomayor encontró publicados on line en los archivos estatales del Ministerio de Educación, Cultura y Deportes de España (AGI. ES.41091.AGI/27.22/MP-PERU- CHILE 264) dos mapas anónimos de 1611 que cubrían el área norte del río Mapocho y que proporcionaban datos sobre las redes viales, entre las cuales destacaban dos caminos del Inga. A partir de esta nueva información se realizaron reconocimientos en terreno que culminaron en la localización precisa de varios segmentos de estos caminos prehispánicos. Este artículo da cuenta de los resultados obtenidos en la investigación.

\section{LOS MAPAS}

Los mapas (AGI. ES.410901. AGI/20.26.2//ESCRIBANIA, 928B) se asocian al litigio por la legalidad de las mercedes de tierras otorgadas en Lampa, entre Juan Ortiz de Araya, Juan de Astorga, Andrés Páez de Aldana, Antonio Recio de Soto y otros, todos vecinos de la ciudad de Santiago. Este litigio debía definir la exacta ubicación de los caminos coloniales e incaicos al norte de la ciudad de Santiago hasta la cuesta de Aconcagua (Chacabuco). Aun cuando los mapas no están a escala y abarcan un amplio espacio, reproducen con bastante exactitud los principales accidentes geográficos y proporcionan detalles que indican un conocimiento minucioso del área. El mapa $\mathrm{N}^{\circ} 1$ tiene por límite norte el cordón de 


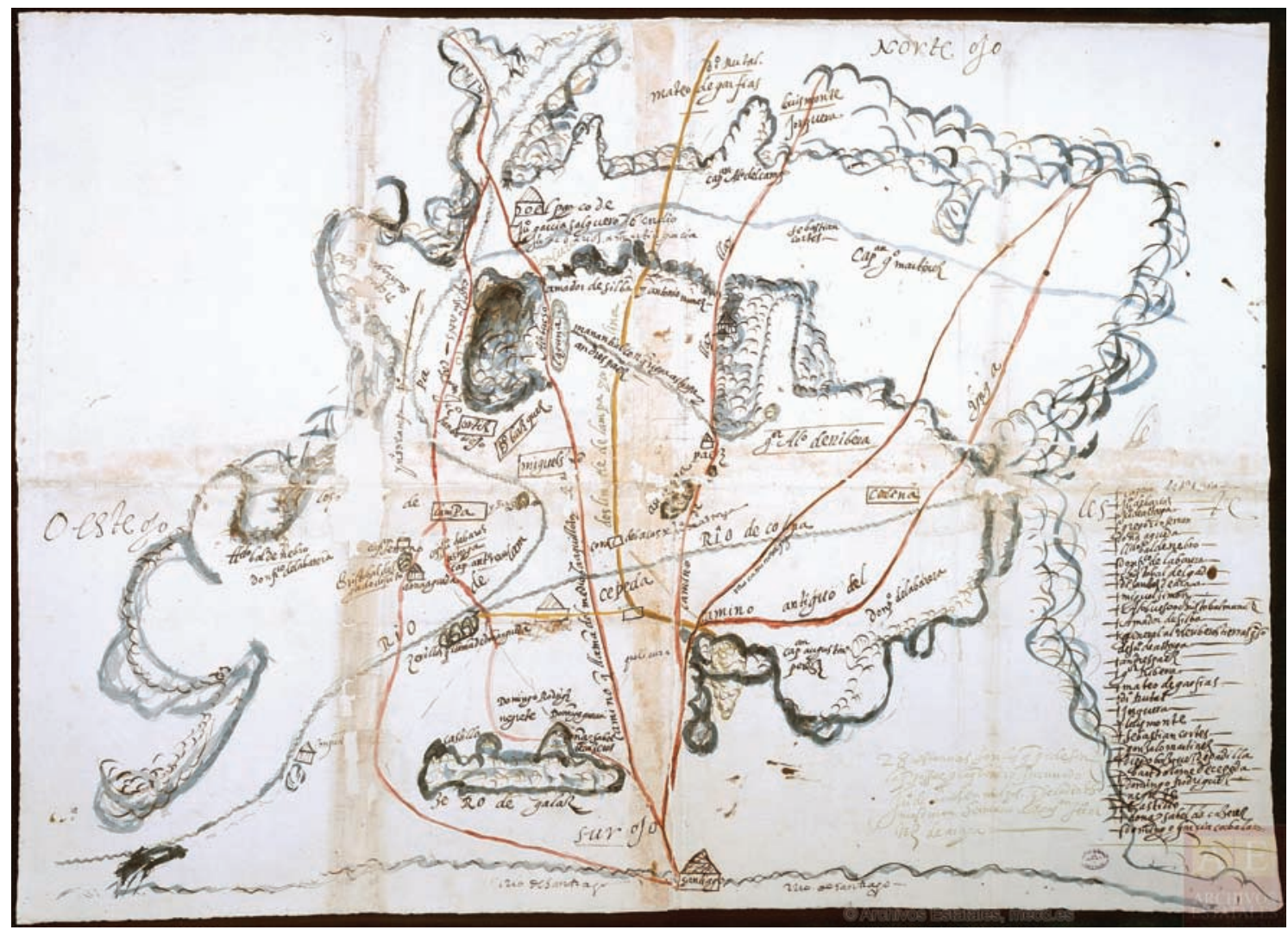

Figura 1. Mapa $\mathrm{N}^{\circ}$ 1, 1611, autor anónimo (AGI. ES.410901. AGI/20.26.2//ESCRIBANIA, 928B). Figure 1. Map Nº 1, 1611, anonymous author (AGI. ES.410901. AGI/20.26.2//ESCRIBANIA, 928B).

Chacabuco; por el sur, el río de Santiago (Mapocho o Mapuche); por el oriente, los primeros contrafuertes de la cordillera andina; $y$ por el poniente, la falda oriental de la cordillera de la costa, incluyendo toda la cuenca norte del Mapocho (fig. 1).

En lo cromático, el mapa utiliza el color negro para definir los nombres de lugares y de personas; el gris para las montañas y cadenas montañosas que servían de límite externo; el café o caoba para los senderos y caminos; el rojo para las rutas de comunicación y el amarillo para señalizar los deslindes de los pueblos de indios.

Los mapas en referencia constituyen una buena oportunidad para tener un acercamiento al paisaje del siglo xvi, al menos de aquellos hitos geográficos que eran importantes para la estructuración del espacio hispano e indígena, como la vialidad, puesto que ese parece ser el foco principal de los planos. En primer lugar, están los tres cordones transversales de cerros que se desprenden de la cordillera andina: el de más al norte corresponde al cordón de Chacabuco, mencionado en el siglo xvi como la "cuesta de Aconcagua"; el del medio corresponde a los cerros de Colina, que nacían en las nacientes del río Colina y casi alcanzaban la cordillera de la costa, a la altura de Lampa; y el de más al sur se originaba a la altura de Chicureo Arriba y se prolongaba hasta el "serro de pan de azúcar", Nigueche (RA 167, f. 194) o Rregueche (RA 167, f. 22), hito de gran importancia simbólica, administrativa y visual tanto para indígenas como para hispanos. Este cordón, luego de una interrupción, continuaba en el cerro de Galáz (actualmente denominado Renca), remarcado en el mapa con una gruesa línea negra.

En segundo lugar, están los portezuelos que atraviesan estas cadenas, que servían de hito vial principal. Nuestros reconocimientos de terreno confirmaron la exactitud de estos puntos y sirvieron para ubicar el trazado de los senderos antiguos, incluyendo los caminos del Inga. 
En tercer lugar, destacan los cerrillos, muy pequeños, pero que en la época eran guías muy valoradas por los transeúntes, entre los cuales destacaban los "zerillos q(ue) llaman de doña agueda” Flores (cerro Calán, RA 167, f. 183v o Calanmaguida, RA 1978, f. 107), lugar donde suponemos existió una posada caminera, por la continua alusión que hacen los viajeros a este sector.

En cuarto lugar, los cursos de agua como el estero de Chacabuco, en la parte superior del mapa, su confluencia con un afluente para formar, por una parte, el río Lampa, y por otra, el río Colina y su juntura con el Lampa. Asimismo, el referido río Mapocho, en la parte inferior del mapa. Destacan, también, los "manantiales de Liray" y la "Laguna” (Tentencura, Batuco), que tenían gran importancia durante el siglo xvi y que hoy están en proceso de desaparición.

El Mapa $\mathrm{N}^{\circ} 1$ presenta una desviación importante respecto del norte geográfico, lo que se evidencia con la palabra "ojo", que el autor colocó al lado de los puntos cardinales sur, oeste y norte. Proporciona, asimismo, la localización de 28 estancias europeas con el nombre de sus propietarios, así como la localización de las tierras de los indios de Colina, Quelicura, Guachuraba y Lampa, con su "tanbo viejo". De acuerdo a documentación inédita registrada por nosotros, este tambo se encontraba en tierras de "chicoco Quilapohe y rrelo" (RA 167, f. 10), mientras que las tierras de los indios de Colina se llamaban de "Comaico e Ilopaique o Lopalque" (RA 2258, fs. 180-183v).

El mapa $\mathrm{N}^{\circ} 2$ es una ampliación del anterior, que se extiende desde el "Serro de Galas", también denominado indistintamente como cerro Renca (RA 1003, fs. 26 y ss) o cerro de Quelicura (RA 167, f. 194) por el sur, hasta el estero y "valle de Chacabuco" por el norte (fig. 2). Muestra con mayor detalle los contrafuertes de la cordillera de la Costa, donde destaca el "Potrero (de los indios) de Lampa" y la "quebrada de Carén". También exhibe con precisión los manantiales de Liray y su drenaje a la "Laguna" (Tentencura. RA 167, f. $68 \mathrm{v}$, hoy Batuco), la cual aparece remarcada con color. Omite mucha información que aparece en el mapa $\mathrm{N}^{\circ}$ 1, como el "tanbo viejo" (Lampa), el "camino antiguo del ynga" y, los deslindes de los pueblos de indios. Por último, enfatiza los senderos coloniales, proporcionando algunas variaciones y agregados respecto al otro mapa.

A juzgar por estos mapas, la importancia estratégica del área fue la conectividad del valle del Mapocho-Maipo con los territorios de más al norte, indispensable para la supervivencia de sus habitantes. En el caso de los contingentes incaicos, las rutas principales iban al pie de la cordillera andina para conectarse, en el curso mediosuperior del valle del Aconcagua, con la ruta trasandina o continuar hacia el norte por las nacientes de los valles de Putaendo, Alicahue, El Sobrante y Choapa. En cambio, los senderos coloniales tempranos se emplazaron más al poniente, en dirección al curso medio y bajo del valle de Aconcagua, lo que evidencia los distintos intereses del Tawantinsuyu y el de los conquistadores españoles.

\section{CAMINOS DEL INGA}

\section{Camino antiguo del ynga}

El mapa $\mathrm{N}^{\circ} 1$ dedica la mitad derecha a mostrar cómo el camino partía de la ciudad de Santiago y se extendía como una línea casi recta, dejando a su mano izquierda las tierras de "quelicura", hasta atravesar un portezuelo (Huechuraba) al pie oriente de un cerro ("Serro de pan de azúcar", Nigueche o Regueche) para bifurcarse en dos ramales paralelos (fig. 1). Uno de ellos, el "camino antiguo del ynga", seguía una dirección oriente hasta llegar a la estancia de "Don j[uan] de la Barrera" y de allí tomaba dirección norte, cruzaba el "Río de Colina", trasponía el portezuelo de los cerros de Colina (Checamo según RA 985 , f. 14) y continuaba recto hasta el cordón transversal de Chacabuco (cuesta de Aconcagua). La denominación de "camino antiguo" alude al origen prehispánico de esta ruta, confirmada por los testimonios documentales que se entregan más adelante.

\section{Otro camino del inga}

El mapa $\mathrm{N}^{\circ} 2$ muestra el ramal de este nombre que se iniciaba en el nodo del portezuelo Guachuraba, seguía al oeste del tambo e iglesia de Colina, teniendo a su izquierda la estancia de Alonso del Pozo, para luego atravesar el cuarto portezuelo de los cerros de Colina, al poniente del anterior (fig. 2). Es interesante que ninguno de estos caminos pasaba por el tambo y pueblo de Colina, como lo sugirieron Stehberg y Carvajal (1988a) y Rivera y Hyslop (1984), lo que se explica por el hecho que este pueblo y su infraestructura física recién se organizó en 1579-1580. Este mapa omite el "camino antiguo del ynga", situación que se explicaría por las discrepancias entre los intereses de los españoles que litigaron por 


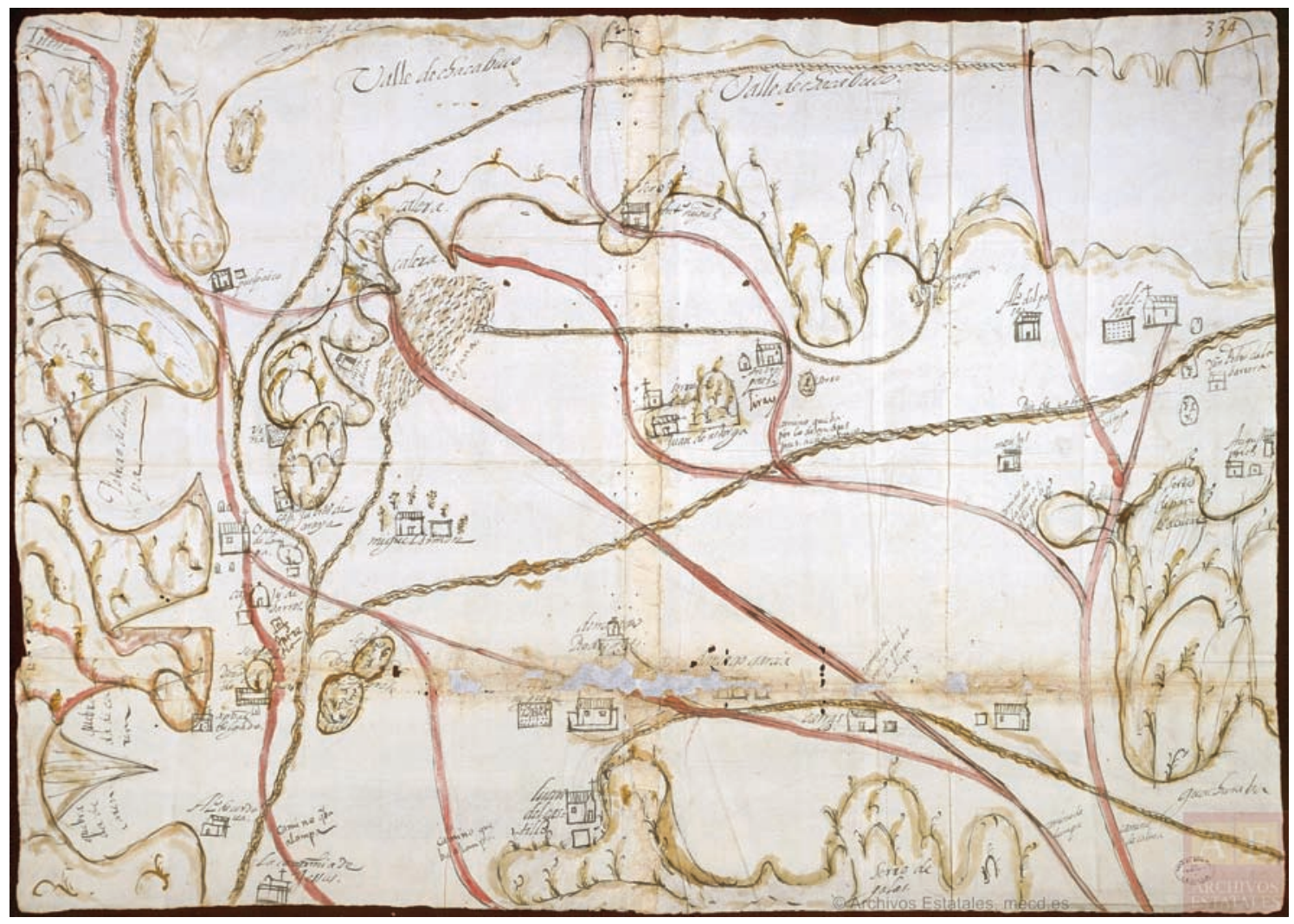

Figura 2. Mapa $\mathrm{N}^{\circ}$ 2, año 1611, autor anónimo, mostrando detalles de Mapocho norte. Figure 2. Map $N^{\circ}$ 2, 1611, anonymous author, depicts details of North Mapocho.

definir cuál camino correspondía al deslinde de sus tierras (RA 1978).

\section{REFERENCIAS DOCUMENTALES AL CAMINO DEL INGA}

Los mapas $\mathrm{N}^{\circ} 1$ y N 2 (AGI. ES.41091. AGI/27.22/MP-PERÚCHILE 264) formaron parte de un extenso juicio colonial de 1611 (RA vol. 1978), cuyo objetivo fue determinar cuál era el verdadero Camino del Inga, el cual servía de deslinde de algunas propiedades europeas, motivo del litigio. Los testigos del juicio declararon conocer el camino del Inga y sus pircas construidas a ambos costados, camino que viniendo del Mapocho pasaba cerca de Colina rumbo a Putaendo y Coquimbo. El indio Gaspar, quechua hablante, natural del Perú, de 60 años aproximadamente, señaló que el valle y las tierras de Lampa se extendía desde "el serro que llaman de Galas hacia la cuesta de Aconcagua que es como ban desta ciudad hacia la d[ic]ha cuesta y ba por el camino rreal del ynga antiguo y ba a salir a Putayendo..." (RA vol. 1978, pza 1, f. 133). Gonzalo, indio del Cuzco, quechua hablante, lo denominó "camino antiguo del ynga que ba e pasa Chacabuco y ba a salir a Putayendo" (RA vol. 1978, pza 1, f. 135).

Cristóbal Muñoz, de más de 60 años, señaló que:

el camino antiguo que llaman de Chile del Ynga pasa por junto a colina e que mas de quarenta y cinco años que este testigo saue que es el $\mathrm{d}(\mathrm{ic})$ ho camino que es por donde los yndios antiguos abian caminado el Ynga a el tiempo que bino a esta tierra e por el caminaban de hordinario antiguamente los que yban y benian a Coquimbo y que (sic) dia estaran las señales del dho camino del qual se rremite por estar señaladas con piedras de un cauo y de otro (RA vol. 1978, pza 1, f. 137).

El Capitán Juan de Córdoba, de aproximadamente 60 años (f. 137v), testificó que "el camino antiguo del Ynga es el que pasa por junto a Colina hacia Aconcagua que oy d[ic]ho dia esta todavía señalado". 
Melchor de Esixa, de más de 83 años (f. 138v), mencionó como deslinde de la estancia de Pedro de Valdivia "el camino rreal del Ynga que es el camino que ba e pasa cerca de Colina por donde en aquel tiempo se solia caminar para yr a Coquimbo y Chuapa y a Putaendo [...] esta asi mismo muy señalado el dia de oy por que estan las piedras puestas". Marcos Griego, vecino morador de Santiago (f. 139v, 140), expresó que "el camino antiguo por donde se solia antiguamente caminar es el camino del Ynga que ba e pasa por junto a Colina que ba a salir a la cuesta de Aconcagua y de alli a Putayendo".

\section{RECONOCIMIENTOS EN TERRENO²}

El objetivo fue identificar en terreno la información proporcionada por los mapas $\mathrm{N}^{\circ} 1$ y $\mathrm{N}^{\circ} 2$. Entre los rasgos del paisaje interesaba localizar los "manantiales de Liray", los cerrillos de "doña Agueda" y otros rasgos señalizados en los mapas. Asimismo, se deseaba determinar la ubicación del mencionado en el mapa como "tanbo viejo" en Lampa y, sobre todo, las rutas seguidas por las principales redes viales del siglo XVI, incluidas los caminos del Inga.

Las primeras salidas se orientaron a conocer el grado de confiabilidad y los criterios utilizados por el autor anónimo de los planos. Pronto se constató que el dibujante conocía muy bien el área. Asimismo, que utilizó como puntos de referencia principalmente los cerros y portezuelos. Justamente estos rasgos fueron los que permitieron localizar con precisión el trazado antiguo de las redes viales.

Respecto a los caminos del Inga, objeto de este artículo, los reconocimientos en terreno se iniciaron, por el sur, en el portezuelo de Huechuraba, al pie oeste del cerro Pan de Azúcar o Negueche. El primer ramal prospectado correspondió al "camino antiguo del ynga". Su primera parte, marcado en el Mapa $\mathrm{N}^{\circ} 1$ como una línea oeste-este, no se pudo identificar. Recién al llegar al actual camino de Los Ingleses (Chicureo), de orientación norte-sur, se tuvo certeza de que la vía correspondía con bastante seguridad a esta ruta. La presencia de algunas piedras a ambos lados del camino apoyó dicha presunción (fig. 3). El camino pasaba por un sector con ocupación del período Tawantinsuyu, como lo evidenció el hallazgo de un jarro decorado con diseños incaicos en el colegio San Anselmo, en el camino Guay Guay del condominio Chicureo Alto, coordenadas 33.267492,
-70.64021 (información gentilmente proporcionada por Roberto Quiroga, 2015); por el hallazgo de un cementerio del período Tawantinsuyu en el condominio Piedra Roja (Westfall 2014, comunicación personal) y de un entierro con cerámica del período Tawantinsuyu en el sitio LвСH-01 (Vargas 2014).

El camino pasaba al pie del cerro Mallautín, mencionado en la documentación colonial como límite étnico entre los pueblos de indios de Chicureo y Colina, para proseguir rectilíneo por el camino Santa Filomena, también denominado camino Coquimbo (es decir, que va a Coquimbo), continuar hasta el pie oeste del cerro la Guaca e inmediatamente atravesar el estero Colina. Cabe mencionar que tanto el camino de Los Ingleses como el camino Coquimbo están siendo ocupados por los condominios que se están construyendo en el área. Hay tramos convertidos en basural. Dado que ya no se puede recorrer íntegramente el camino, se debe hacer grandes rodeos para retomar los tramos cortados.

Tanto los informantes locales como la cartografía antigua señalan que el camino de Los Ingleses y su continuación como camino Coquimbo era el único camino que atravesaba longitudinalmente el sector. Los restos de una antigua casa con pilar de piedra en San Luis, seguramente de fines del siglo XviII, confirmaron la antigüedad colonial de la ruta, mientras que la presencia continua de piedras a ambos lados del camino sugirió su origen incaico, concordante con la documentación colonial temprana que señalaba que el camino antiguo iba amurallado por ambos costados.

A los 31,8 km al norte de Santiago, la ruta pasó al pie del cerro La Guaca, cruzó el estero Colina y penetró en un vallecito irrigado por el estero Colina, donde existió un asentamiento de la Cultura Aconcagua, con presencia de sepulturas incaicas, lo que se interpretó como intervención Tawantinsuyu de un espacio simbólico local (Hermosilla et al. 2005). En este sector, la ruta incaica cruzó diagonalmente el pueblo actual de Esmeralda, rompiendo su trazado ortogonal, para ascender directo al primer portezuelo de los cerros de Colina, tal como lo señalara el Mapa $\mathrm{N}^{\circ} 1$. Continuaba rumbo norte, por un extenso tramo que no pudo ser identificado, hasta unirse al otro ramal cerca del cordón de Chacabuco y atravesar como uno solo por la cuesta de Aconcagua. Este sector estuvo sacralizado mediante la representación de un $u n k u$ ajedrezado en la caverna Iglesia de Piedra que personificaba al Inca o su ejército (Berenguer 2011). 




Figura 3. Reconocimiento del camino de Los Ingleses, en Chicureo-Colina, coincidente con el trazado del "camino antiguo del Inga". Nótese las piedras a ambos lados del camino y como el camino está desapareciendo por urbanización. Figure 3. Referential image of "Los Ingleses road", in Chicureo-Colina, matching the trace of the "camino antiguo del Inga" ('Inca's ancient road'). Notice the stones at both sides of the road and how the path is currently disappearing because of urbanization.

La ruta del "otro camino del inga" corresponde muy posiblemente a una calle ancha, situada inmediatamente al oeste del portezuelo Huechuraba, de dirección norte, sin salida, convertida en basural, que termina en una propiedad agrícola particular. A partir de ese punto, no se pudo continuar el reconocimiento. Tras un largo rodeo, se llegó a la calle La Capilla, que se extiende a unos kilómetros al oeste del actual pueblo de Colina y que se dirige directamente al cuarto portezuelo de los cerros de Colina, concordante con los datos proporcionados por el Mapa $\mathrm{N}^{\circ} 1$. A partir de ese punto el trazado, coincidió con el antiguo camino vehicular empedrado a la ciudad de Los Andes, que estuvo en uso hasta la segunda mitad del siglo xx. Continúa por terrenos de Minera Los Bronces, en Peldehue, con presencia de restos de muros de piedra a ambos costados de la vía (fig. 4). Finalmente, el camino confluye en la actual carretera internacional a Los Andes y Mendoza, continuando por este mismo trazado ruta hasta la cuesta de Aconcagua. Según el mapa $\mathrm{N}^{\circ} 1$, los dos caminos incaicos proseguían paralelos hasta penetrar en el cordón de Chacabuco, situación que no se pudo comprobar en terreno. La fig. 5 ilustra los trazados de las vialidades del siglo xvi y comienzos del siguiente, tanto incaicos como coloniales, reconstruidos a través del análisis cartográfico, histórico $y$ de terreno efectuados en este estudio.

\section{DISCUSIÓN Y CONCLUSIONES}

El hallazgo de estos dos mapas en 2013 nos proporcionó una gran oportunidad para avanzar en el conocimiento del trazado del Qhapaq Nan en el tramo Mapocho norte, para entender su relación con el extenso juicio colonial 


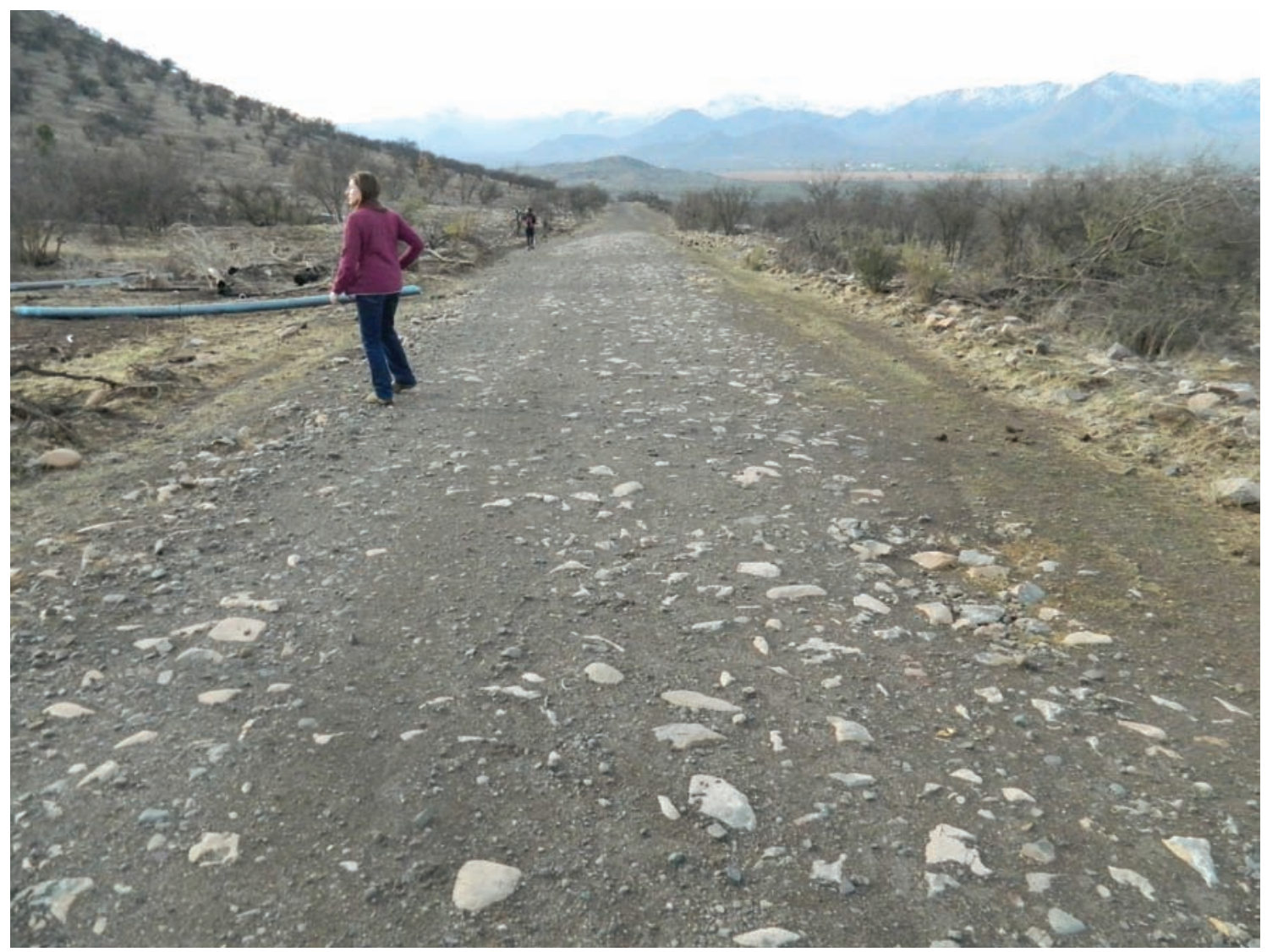

Figura 4. En la foto se observa restos del antiguo camino vehicular a Los Andes, en el sector de Peldehue, construido sobre el "otro camino del Inga". Figure 4. The photograph shows the remains of the old vehicular road to Los Andes in the Peldehue sector, built on the "other way of Inga".

de 1611 y las declaraciones discrepantes presentadas por alguno de sus testigos.

Hasta esa fecha, la mayoría de los especialistas suponía que la zona norte de Santiago era atravesada longitudinalmente por un solo camino incaico, el cual pasaba al costado oeste de la antigua iglesia de Colina (Rivera \& Hyslop 1984; Stehberg 1995). Sin embargo, el Mapa $\mathrm{N}^{\circ} 1$ mostró la existencia de dos caminos incaicos paralelos y que ninguno pasaba por el lugar donde estuvo el tambo e iglesia de Colina. El "camino antiguo del ynga" circuló a varios kilómetros al este de este pueblo, mientras que el "otro camino del ynga" lo hizo a varios kilómetros al oeste.

Gracias al título con el que Pedro de Valdivia le entregó a Marcos Veas la mitad del valle y la mitad de los indios de Lampa, se sabe que el límite oriente de la "estancia del Gobernador" era el camino del Inga (RA vol. 167, 686 y 1978). Habiendo dos caminos paralelos incaicos, surgió el problema de su deslinde entre los herederos de Marcos Veas y sus vecinos. Este inconveniente desembocó en el pleito de 1611 y en la elaboración de los mapas presentados en este artículo.

Es destacable el paralelismo de estos dos caminos, práctica bastante común en el proceso de construcción y ritualización del paisaje por parte del Tawantinsuyu (Vitry 2001, 2007; Martínez 2009; Acuto 2014). Su presencia en el Mapocho norte reafirma la importancia que tuvo esta área para el Tawantinsuyu.

A partir de los datos disponibles, se proponen algunas hipótesis a ser confrontadas en el futuro, cuando se disponga de mayores antecedentes. Una de ellas surge de la observación de la parte sur del "camino antiguo del ynga”, tal como aparece en el Mapa $\mathrm{N}^{\circ} 1$. Allí, el camino tuerce hacia el poniente para conectarse con el "otro camino del ynga" y atravesar juntos el portezuelo de Huechuraba rumbo a Santiago. En esta oportunidad, 


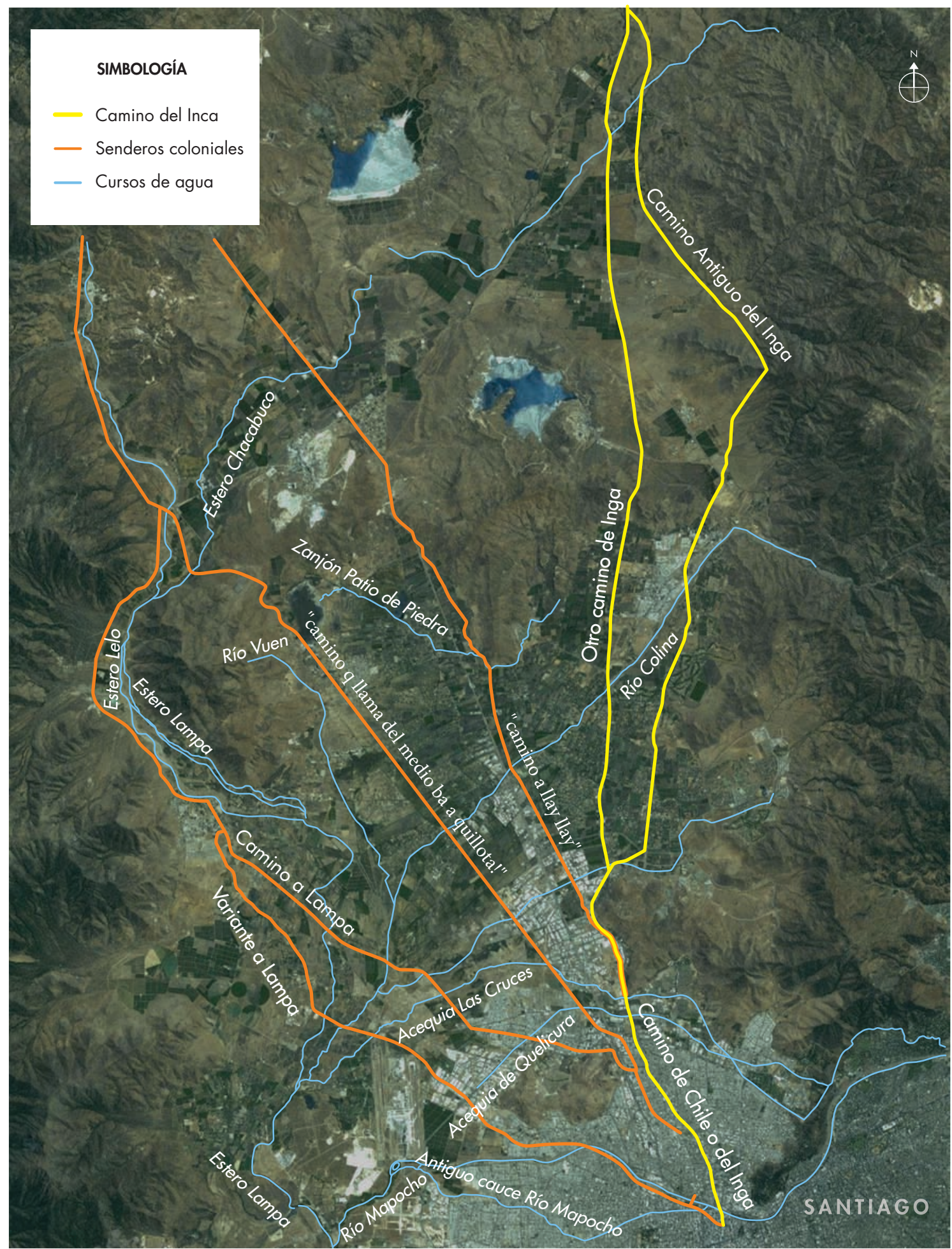

Figura 5. Mapa con la localización de las rutas viales incaicas y coloniales utilizadas durante el siglo xvi y comienzos del siguiente, de acuerdo a los Mapas $\mathrm{N}^{\circ} 1$ y N 2 y luego de nuestros reconocimientos en terreno. Figure 5. Map showing Inca and Colonial roadways that were used during the 16th and early 17th centuries, according to the Maps $N^{\circ} 1$ and $N^{\circ} 2$, and to our fieldwork analysis. 
se propone que el "camino antiguo del ynga" continuó rumbo sur-oriente para cruzar una cadena de cerros y penetrar en el sector de la Dehesa, luego cruzar el río Mapocho y continuar por los faldeos de la cordillera de Apoquindo, Peñalolén y Macul, en dirección al río Maipo y portezuelo de Chada, en el cordón de Angostura.

Durante el período Tawantinsuyu, las tierras de Apoquindo, Peñalolén y Ñunoa fueron dedicadas a la agricultura, siendo alimentadas por la acequia antigua de Tobalaba y la acequia de Macul (Stehberg \& Sotomayor 2012). En este sentido, se postula que el paralelismo de los caminos incaicos tenía una mayor extensión, ya que no concluía en el portezuelo de Huechuraba, como indica el Mapa $\mathrm{N}^{\circ}$, sino que atravesaba toda la cuenca del Mapocho-Maipo, situación que ya fue sugerida en la publicación antes mencionada (Stehberg \& Sotomayor 2012). Esta hipótesis requiere de mayores estudios futuros.

La segunda hipótesis se relaciona con la posible existencia de instalaciones arquitectónicas (tambos, chasquiwasi) asociadas a los caminos que se están describiendo, las que han desaparecido por problemas asociados al crecimiento urbano y agrícola de Santiago. La primera debió estar en el sector del portezuelo Huechuraba, distante $16,3 \mathrm{~km}$ al norte del propuesto centro administrativo y ceremonial del Mapocho (Stehberg \& Sotomayor 2012). En dicho sector, el "serro de pan de azúcar" (Negueche) fue un apu que sirvió de orientación a los transeúntes debido a su alta visibilidad desde diferentes puntos del valle del Mapocho.

El portezuelo fue mencionado reiteradamente por los testigos del juicio de 1611 como el lugar exacto por donde pasaba el camino del Inga (ACSD vol. 18). Respecto al sector del nodo vial, Tomás indio declaró: "vido venir el dho Inga, y le conoció, y a su jente, y le vido, que en la falda de dho serro del portezuelo abaxo hicieron fuego los Yndios Ingas, y pasaron por mitad de las tierras y casa que ahora tiene pobladas en su chacara el dho Juan Peres de Caceres" (ACSD vol. D 18, f. 8), declaración que sugiere que allí existió una instalación. A mayor abundamiento, el Mapa $\mathrm{N}^{\circ} 1$ señala que el deslinde entre los pueblos de indios de Colina y Quelicura pasó justamente por allí, demarcación que seguramente tiene antecedentes en tiempos prehispánicos anteriores.

La siguiente instalación arquitectónica asociada al camino debió estar 15,8 km más al norte, en el sector de un nucleamiento incaico emplazado en las inmediaciones del sitio Peldehue (Hermosilla et al. 2005), próximo al cerro La Guaca. Fue este el sector que escogieron los indios del pueblo de Colina cuando, en 1603, el agrimensor Ginés de Lillo les consultó dónde querían que se les asignaran tierras; sector que refirieron como junto al camino del Inga.

La siguiente instalación vial (tambo) debió emplazarse en el sector de casas de Chacabuco, 18,3 km más al norte del anterior (46 km al norte de Santiago) -lugar donde el arqueólogo Hans Niemeyer encontró fragmentos de cerámica del período Tawantinsuyu- y, finalmente, en El Castillo ( $60 \mathrm{~km}$ al norte de Santiago), en el valle de Aconcagua, punto nodal del camino inca longitudinal y el camino inca trasandino.

Con respecto al ramal paralelo, llama la atención que no se ha encontrado evidencia arqueológica asociada, lo que sumado a que el mapa $\mathrm{N}^{\circ} 1$ lo denomina "otro camino del Inga", sin el adjetivo "antiguo", hace pensar en una obra más reciente, incluso contemporánea a la primera ocupación hispana.

El área que se extiende al oeste del "otro camino del Inga" posee casi nula presencia Tawantinsuyu, interrumpida exclusivamente por el sitio Huechún 3 (Stehberg 1981). Este es un importante asentamiento de la Cultura Aconcagua que tuvo fuerte contacto incaico. En el valle de Lampa, por el contrario, se desarrolló otro sector de nucleamiento indígena con fuerte representación Tawantinsuyu. Allí, aparentemente, el Tawantinsuyu no construyó nuevos caminos, sino que aprovechó los antiguos senderos de la población local, los cuales, por lo demás, siguieron en uso durante el período colonial temprano. En este valle, el asiento principal estuvo en "tanbo viejo", desde el cual, postulamos, se administró la extensa población local incaizada, que dejó testimonio material en distintos lugares a lo largo del valle (Thomas et al. 1989) y una colonia mitimae de indios "paxareros" en los alrededores de la Laguna, hoy Batuco (Stehberg \& Sotomayor 2012).

Otro sector de nucleamiento incaico se localizó en el sector de Quilicura, al sur del valle de Lampa y a medio camino hacia el centro administrativo del Mapocho, con importante presencia de sepulturas del período Tawantinsuyu. Este sector fue incorporado al riego mediante la acequia Quilicura procedente de El Salto, de origen incaico.

Finalmente, el sector de nucleamiento Tawantinsuyu de Conchalí, Guachuraba y Renca fue regado por la "acequia madre de Guachuraba", de igual origen, lo que permitió el amplio desarrollo de la agricultura. Vestigios de sus pobladores se han reconocido en varios hallazgos de sepulturas del período considerado. 
En general, se constató una ocupación Tawantinsuyu de espacios definidos para control de rutas y de explotación agrícola y productos suntuarios (plumas), donde la estrategia no era coercitiva (no existen pucarás o reductos fortificados en el área), sino de "conquista ritual" (Hermosilla et al. 2005) o de introducción de "elementos de eficacia simbólica" (Uribe 1999-2000). Aparentemente, la población local se incorporó pacíficamente al Tawantinsuyu, manteniendo parte de sus manifestaciones culturales locales. En el sitio habitacional de Huechún-3, por ejemplo, los platos de cerámica tipo Aconcagua Anaranjado mantuvieron en su cara exterior el emblemático motivo trinacrio en color negro sobre naranja, mientras que la superficie interior fue pintada con diseños trícromos de origen cuzqueño o diaguitaincaico. En el cementerio Las Tinajas, en Quilicura, los grupos locales acomodaron y resignificaron los diseños con motivos foráneos traídos por el Tawantinsuyu, adaptándolos a su cosmovisión. Así, por ejemplo, motivos decorativos cerámicos que en el área diaguita o cuzqueña eran organizados espacialmente siguiendo el principio de la cuatripartición, en el Mapocho fueron agrupados siguiendo el principio de la tripartición. Resulta bastante evidente que, con el apoyo y la colaboración de la comunidad local, el Tawantinsuyu pudo controlar el estratégico acceso norte de la cuenca del Mapocho.

En este sentido, la estrategia seguida por el conquistador Pedro de Valdivia y sus soldados fue bastante distinta. La convivencia pacífica con los habitantes del Mapocho-Maipo duró muy poco y al cabo de unos meses los enfrentamientos se hicieron cada vez más intensos (León 1991). Valdivia, consciente de la importancia de mantener el control de la población local, de las vías de comunicación marítima y terrestre con el Perú y del acceso a recursos naturales valiosos como el oro de Tiltil, la cal de Tentencura y los bosques aledaños, se autoasignó el territorio comprendido entre el cerro de Galaz por el sur y el río Aconcagua por el norte, y desde el Qhapaq Nan por el oriente hasta las tierras bajas cercanas al océano por el poniente, lo que incluyó a sus habitantes que, en la práctica y pese a la legislación protectora emanada de la Corona a favor de los indígenas, pasaron a ser esclavos. Constituyó así una unidad económica que se conoció como la "Estancia del Gobernador". En su interior se impuso una férrea disciplina conforme a la cual, por ejemplo, "se le cortó un pie" al cacique principal del valle de Lampa por quererse rebelar (RA vol. 1978, pieza $1^{\text {a }}$ ). Controlada la región hacia 1552 , cedió sus tierras a terceros, partiendo por la mitad el valle de Lampa con la mitad de sus indios, que fueron entregados el 14 de noviembre de 1552. Posteriormente, el Cabildo de Santiago continuó otorgando estas tierras a beneméritos del Reino, para estancia de su ganado.

Un proceso distinto siguieron las bien irrigadas tierras de Conchalí, Huechuraba, Renca y Quilicura, las cuales, presumiblemente en virtud de la creencia de ser incaicas, fueron tempranamente otorgadas en merced de chacra a los vecinos europeos de la ciudad de Santiago para vivienda y cultivo, proceso similar al resto de las tierras dadas en chacra de otros sectores de la cuenca de los ríos Maipo-Mapocho.

Los mapas en referencia confirmaron la importancia estratégica de esta área, representada por su conectividad con los territorios de más al norte, donde los senderos fueron indispensables para la supervivencia de los habitantes de los valles de Mapocho-Maipo, tanto en tiempos prehispánicos tardíos como durante el proceso de conquista europea. De las cinco rutas viales en uso durante el siglo xvi y principios del siguiente, dos tuvieron origen incaico, y de las tres restantes, al menos una es mencionada como de origen indígena local.

\section{NOTAS}

${ }^{1}$ Estos trabajos se hicieron en el marco del proyecto "Articulaciones entre instalaciones arquitectónicas, red hidráulica, caminos estatales y paisaje ritualizado en los valles de MapochoMaipo durante el período Tawantinsuyu", patrocinado por el Museo Nacional de Historia Natural y financiado por FONDECYT 1140043/2014-2016.

${ }^{2}$ Se realizaron diez salidas a terreno los meses de junio y julio de 2014, en las cuales participaron los autores de este artículo más el arqueólogo José Berenguer.

\section{REFERENCIAS}

Acuto, F., 2014. Paisajes rituales incaicos. Una mirada desde las crónicas coloniales. Haucaypata 3 (8): 123-130. Lima.

Berenguer, J., 2011. Unkus ajedrezados en el arte rupestre del sur del Tawantinsuyu: ¿la estrecha camiseta de la nueva servidumbre? En Las tierras altas del área centro sur andina entre el 1000 y el 1600 D.C. TANOA II, M. Albeck, M. Ruiz \& B. Cremonte, Eds., pp. 311-352. Jujuy: Universidad Nacional de Jujuy.

Hermosilla, N.; C. González \& D. Baudet, 2005. Sitio Peldehue: rescate de un contexto funerario Inca en un sitio habitacional Aconcagua. Xama 15-18: 263-278. 
Keller, C., 1960. Los orígenes de Quillota. Boletín Academia Chilena de la Historia. Santiago.

LEón, L., 1991. La merma de la sociedad indígena de Chile central y la última guerra de los Promaucaes, 1541-1558. St. Andrews: Institut of Ameridian Studies, University of St. Andrews.

MartínEZ, G., 2009. Qhapaq Ñan. El Camino Inca y las transformaciones territoriales en los Andes peruanos. Ería 78-79: 21-38.

Mostny, G., 1947. Un cementerio incásico en Chile Central. Boletín del Museo Nacional de Historia Natural 23: 17-41.

Mostny, G., 1957. La Momia del Cerro El Plomo. Boletín del Museo Nacional de Historia natural 27 (1).

Rivera, M. \& J. Hyslop, 1984. Algunas estrategias para el estudio del Camino del Inka en la Región de Santiago. Cuadernos de Historia 4: 109-112.

Rosales, A., 1887. La Cañadilla de Santiago. Su historia i sus tradiciones. 1541-1887. Santiago: Establecimiento tipográfico de La Época.

Rosales, A., 1948. La Chimba antigua. La Cañadilla de Santiago (1541-1887). Colección Letras Chilenas. Santiago: Difusión.

Silva, O., 1977-1978. Consideraciones acerca del Período Inca en la cuenca de Santiago (Chile central). Boletín del Museo Arqueológico La Serena 16: 211-243.

Stehberg, R., 1976. La fortaleza de Chena y su relación con la ocupación incaica de Chile central. Publicación Ocasional del Museo Nacional de Historia Natural (Chile) 23: 3-37.

Stehberg, R., 1981. El complejo prehispánico Aconcagua en la Rinconada de Huechún. Publicación Ocasional del Museo Nacional de Historia Natural 35.

Stehberg, R., 1995. Instalaciones incaicas en el norte y centro semiárido de Chile. Santiago: Centro de Investigaciones Diego Barros Arana-DIBAM.

Stehberg, R.; N. Carvajal \& R. Seguel, 1986. El Tambo Conchuca y su relación con la ruta de penetración inka al centro de Chile. Comechingonia 4: 15-42.
Stehberg, R. \& N. Carvajal, 1988a. Red vial incaica en los términos meridionales del Imperio: tramo valle del Limarí-valle del Maipo. BAR International series 442: 181-214.

Stehberg, R. \& N. Carvajal, 1988b. Road system of the Incas in the southern part of their Tawantinsuyu Empire. National Geographic Research 4 (1): 74-87.

Stehberg, R. \& G. Sotomayor, 2012. Mapocho Incaico. Boletín del Museo Nacional de Historia Natural 61: 85-149.

Thomas, C.; A. Benavente, C. Massone, R. Isaurieta, D. Jackson, A. Murga, R. Sánchez, I. Cartajena \& C. Becker, 1989. Arqueología de la comuna de Lampa. Informe final proyecto FONDECYT 1240-88. Santiago.

Uribe, M., 1999-2000. La arqueología del Inka en Chile. Revista Chilena de Antropología 15: 63-97.

VARGAS, F., 2014. Salvataje arqueológico Entierro 1. Sitio LBCH-01. Condominio Las Brisas de Chicureo, Colina, RM. Informe para el Consejo de Monumentos Nacionales.

Vitry, CH., 2001. Los Inkas y el paisaje. Organización geopolítica y religiosa del territorio prehispánico. Conferencia presentada en el III Encuentro Internacional Alexander Von Humboldt, 15 al 19 de octubre de 2001. Salta, <https:// www.academia.edu/4088464/Los_Inkas_y_el_paisaje._ Organizaci\%C3\%B3n_geopol\%C3\%ADtica_y_religiosa_del_territorio_prehisp\%C3\%A1nico> [Consultado 19-04-2017].

VITRY, CH., 2007. Caminos rituales y montañas sagradas. Estudio de vialidad inka en el nevado de Chañi, Argentina. Boletín del Museo Chileno de Arte Precolombino 12 (2): 69-84. 